13,05

\title{
Экспериментальное исследование коэффициента теплопроводности в тонких пленках на основе одностенных углеродных нанотрубок
}

\author{
(C) И.А. Тамбасов ${ }^{1}$, А.С. Воронин ${ }^{2,5}$, Н.П. Евсевская ${ }^{3}$, Ю.М. Кузнецов ${ }^{4}$, А.В. Лукьяненко ${ }^{1,5}$, \\ Е.В. Тамбасова ${ }^{6}$, М.О. Горнаков ${ }^{1}$, М.В. Дорохин ${ }^{4}$, Ю.Ю. Логинов ${ }^{6}$ \\ ${ }^{1}$ Институт фризики им. Л.В. Киренского СО РАН, ФИЦ КНЦ СО РАН, \\ Красноярск, Россия \\ ${ }^{2}$ Красноярский научный центр СО РАН, ФИЦ КНЦ СО РАН, \\ Красноярск, Россия \\ ${ }^{3}$ Институт химии и химической технологии СО РАН, ФИЦ КНЦ СО РАН, \\ Красноярск, Россия \\ ${ }^{4}$ Научно-исследовательский фризико-технический институт \\ Нижегородского государственного университета им. Н.И. Лобачевского, \\ Нижний Новгород, Россия \\ ${ }^{5}$ Сибирский федеральный университет, \\ Красноярск, Россия \\ ${ }^{6}$ Сибирский государственный университет науки и технологий им. М.Ф. Решетнева, \\ Красноярск, Россия \\ E-mail: tambasov_igor@mail.ru
}

Поступила в Редакцию 11 ноября 2019 г.

В окончательной редакции 21 января 2020 г.

Принята к публикации 21 января 2020 г.

\begin{abstract}
Тонкие пленки на основе одностенных углеродных нанотрубок с толщиной от $11 \pm 3$ до $157 \pm 18 \mathrm{~nm}$ были сформированы с помощью вакуумной фильтрации. Коэффициент теплопроводности в тонких пленках был исследован в зависимости от толщины и температуры до $450 \mathrm{~K}$ с помощью $3 \omega$-метода. Обнаружено, что в области $49 \mathrm{~nm}$ подведенное тепло от золотой полоски начинало эффективно распространяться в плоскость тонкой пленки. Коэффициент теплопроводности для тонких пленок с толщиной от $49 \pm 8 \mathrm{~nm}$ был измерен согласно $3 \omega$-метода для объемных образцов. Было обнаружено, что коэффициент теплопроводности в тонких пленках на основе одностенных углеродных нанотрубок сильно зависит от толщины и температуры. Коэффициент теплопроводности резко повышался ( 60 раз) при увеличении толщины с $11 \pm 3$ до $65 \pm 4 \mathrm{~nm}$. Кроме этого, было выявлено, что коэффициент теплопроводности для $157 \pm 18 \mathrm{~nm}$ тонкой пленки стремительно уменьшался с $211 \pm 11$ до $27.5 \pm 1.4 \mathrm{~W} \cdot \mathrm{m}^{-1} \cdot \mathrm{K}^{-1}$ для 300 и $450 \mathrm{~K}$ соответственно.
\end{abstract}

Ключевые слова: одностенные углеродные нанотрубки, вакуумная фильтрация, тонкие пленки, коэффициент теплопроводности.

DOI: $10.21883 /$ FTT.2020.06.49358.625

\section{1. Введение}

Ключевыми материалами для гибкой электроники являются различные аллотропные формы углерода $[1,2]$. С точки зрения разработки и создания реальных прототипов гибкой электроники перспективными являются одностенные углеродные нанотрубки (ОУНТ) [1,3]. Для гибкой электроники, в том числе для гибких термоэлектрических преобразователей [4] на основе ОУНТ, необходимо знать механизм теплопереноса. Перенос тепла в системах на основе ОУНТ достаточно сложный и зависит от сформированной структуры системы. Механизм переноса тепла в единичных одностенных и многостенных углеродных нанотрубках имеет фононную природу [5]. В случае, когда длина свободного пробега фонона больше, чем длина нанотрубки, имеет место баллистический фононный транспорт [6]. Основным каналом диссипации является фонон-фононное рассеяние на дефектах кристаллической решетки. В большинстве прикладных задач используются тонкие пленки на основе именно одностенных углеродных нанотрубок [1-3,7]. В пленках появляется новый эффективный канал рассеяния, связанный с контактами между нанотрубками. В обзорных работах $[8,9]$ было показано, что коэффициент теплопроводности в структурах на основе углеродных нанотрубок может изменяться от 0.1 до $6600 \mathrm{~W} \cdot \mathrm{m}^{-1} \cdot \mathrm{K}^{-1}$. Таким образом, экспериментальные исследования коэффициента теплопроводности в пленочных системах на основе ОУНТ, в зависимости от толщины пленки и геометрических характеристик отдельных нанотрубок (длина, диаметр), являются ключевыми задачами.

В настоящей работе впервые представлены измерения коэффициента теплопроводности в тонких пленках на основе промышленно доступных ОУНТ с помощью $3 \omega$-метода. Сильная зависимость коэффициента теплопроводности от толщины и температуры была обнаружена. 


\section{2. Методика получения и исследования пленок}

Для приготовления водных дисперсий было использовано сухое сырье ОУНТ (Tuball, OCSiAl) и ПАВ холат натрия ( $2 \%$ весовых в водной дисперсии). Такие одностенные углеродные нанотрубки, согласно предыдущим исследованиям $[3,10]$, имели диаметр в диапазоне $\sim 1.6-1.9 \mathrm{~nm}$. Тонкие пленки на основе ОУНТ были получены методом вакуумной фильтрации. Подробное описание методики получения дисперсий и тонких пленок на основе ОУНТ можно найти в работе [10]. В качестве подложки использовалось дисплейное стекло производства AGC. Толщина пленок ОУНТ определялась методом просвечивающей электронной микроскопии (электронный микроскоп Hitachi TM7700) в режиме съемки поперечного сечения $[10,11]$. Здесь стоит отметить, что тонкие пленки на основе углеродных нанотрубок были неоднородны по толщине [10]. Для каждого образца по изображению поперечного сечения было проведено 10 замеров для анализа толщины пленки. Толщина тонкой пленки определялась как среднее арифметическое из 10 замеров со среднеквадратичным отклонением. Таким образом, в настоящей работе исследовались пленки ОУНТ с толщинами $11 \pm 3,34 \pm 12,49 \pm 8,65 \pm 4$ и $157 \pm 18 \mathrm{~nm}$ соответственно. Морфология поверхности тонких пленок определялась с помощью атомно-силового микроскопа DPN 5000 производства Nanoink.

Для измерения коэффициента теплопроводности в тонких ОУНТ пленках использовался $3 \omega$-метод. Для этой цели на каждой пленке ОУНТ были сформированы золотые полоски с помощью оптической фотолитографии. Длина каждой золотой полоски была $3 \mathrm{~mm}$, ширина $20 \mu \mathrm{m}$ и толщина $40 \mathrm{~nm}$. Диэлектрический слой $\mathrm{Al}_{2} \mathrm{O}_{3}$ между тонкой ОУНТ-пленкой и золотой плоской использовался для обеспечения электрической изоляции. Толщина слоя $\mathrm{Al}_{2} \mathrm{O}_{3}$ была $\sim 7 \mathrm{~nm}$. Такая толщина $\mathrm{Al}_{2} \mathrm{O}_{3}$ обеспечивала электрическую изоляцию и вносила минимальную погрешность в величину коэффициента теплопроводности измеряемой тонкой пленки. Слои золото и $\mathrm{Al}_{2} \mathrm{O}_{3}$ на стеклянной подложке и тонких ОУНТ-пленках были получены с помощью электроннолучевого осаждения в одном технологическом процессе. Далее будет подразумеваться, что при измерении частотной зависимости подложки измеряется по сути система слой $\mathrm{Al}_{2} \mathrm{O}_{3}(7 \mathrm{~nm}) /$ стеклянная подложка.

Источник-измеритель Keithley 6221 использовался для задания переменного тока, который протекал через золотую полоску согласно методике $3 \omega$. Селективный вольтметр SR810 Stanford Research System был использован для измерения амплитуды генерируемой 3 гармоники. Измерения коэффициента теплопроводности в тонких ОУНТ пленках были проведены в температурном диапазоне $300-450 \mathrm{~K}$. Все измерения проводились в вакууме с остаточным давлением менее 3 Pa.

Исследование коэффициента теплопроводности в тонких пленках имеет свои особенности. В рамках $3 \omega$-мето- да были проведены измерения $U_{3 \omega}$ от $\operatorname{Ln}(2 \omega)$-подложки, где $U_{3 \omega}$ - амплитуда генерируемого напряжения на утроенной частоте, а $\omega-$ частота переменного тока, подаваемого на золотую полоску. Далее были проведены измерения $U_{3 \omega}$ от $\operatorname{Ln}(2 \omega)$ системы пленка/подложка. Из зависимостей $U_{3 \omega}$ от $\operatorname{Ln}(2 \omega)$ находилась линейная часть. После этого была сделана линейная аппроксимация для этих двух зависимостей. Разница между константами системы пленка/подложка и подложка, полученных при аппроксимации зависимостей $U_{3 \omega}$ от $\operatorname{Ln}(2 \omega)$ в линейной части, определяет коэффициент теплопроводности тонкой пленки. Согласно $3 \omega$-методике [12] коэффициент теплопроводности $\kappa$ для тонкой пленки выделяется как частотно независимое слагаемое

$$
\begin{gathered}
\Delta T_{\text {film }}=\frac{2 U_{3 \omega \text { film } / \text { substrate }}}{I_{\omega \text { film } / \text { substrate }} R_{2} \alpha}-\frac{2 U_{3 \omega \text { substrate }}}{I_{\omega \text { substrate }} R_{1} \alpha}=\frac{P t}{(l \kappa 2 b)}, \\
\kappa=\frac{P t}{\left(\Delta T_{\text {film }} l 2 b\right)},
\end{gathered}
$$

где $U_{3 \omega}$ film/substrate - амплитуда напряжения на утроенной частоте в системе пленка/подложка; $I_{\omega}$ film/substrate амплитуда тока на основной частоте в системе пленка/подложка; $\alpha-$ температурный коэффициент электрического сопротивления золотой полоски; $R 1$ и $R 2-$ электрические сопротивления золотых полосок, используемых для измерения подложки и системы пленка/подложка; $U_{3 \omega}$ substrate амплитуда напряжения на утроенной частоте при измерении подложки; $I_{\omega}$ substrate амплитуда тока на основной частоте при измерении подложки; $P$ - мощность, подаваемая на золотую полоску; $t-$ толщина исследуемой пленки; $\kappa_{\text {film }}-$ коэффициент теплопроводности тонкой пленки, $l$ и $b-$ длина и полуширина золотой полоски соответственно. По сути, тонкая пленка выступает как добавочное термическое сопротивление [12]. Главным условием измерения является одинаковый разогрев подложки и системы пленка/подложка. Для этой цели подбираются необходимые токи на основной гармонике исходя из сопротивлений золотых полосок и величины $\alpha$. Таким образом, при одинаковом разогреве, зависимость $U_{3 \omega}(\operatorname{Ln}(2 \omega))$ для системы пленка/подложка будет параллельна зависимости $U_{3 \omega}(\operatorname{Ln}(2 \omega))$ для подложки.

Если тепловой поток от золотой полоски распространяется вглубь на расстояние меньше, чем толщина пленки, то тогда используется подход для измерения объемных образцов согласно $3 \omega$ метода [13]. В этом случае коэффициент теплопроводности пленки будет определяться по следующей формуле:

$$
\kappa=-\frac{I_{\omega \text { film/substrate }}^{3} R_{2}^{2} \alpha}{4 l \pi} \frac{\partial \ln (2 \omega)}{\partial U_{3 \omega}},
$$

где $\partial \ln (2 \omega) / \partial U_{3 \omega}$ является обратной величиной углового коэффициента линейной части функции $U_{3 \omega}(\operatorname{Ln}(2 \omega))$. Угловой коэффициент находится также с помощью линейной аппроксимации. 

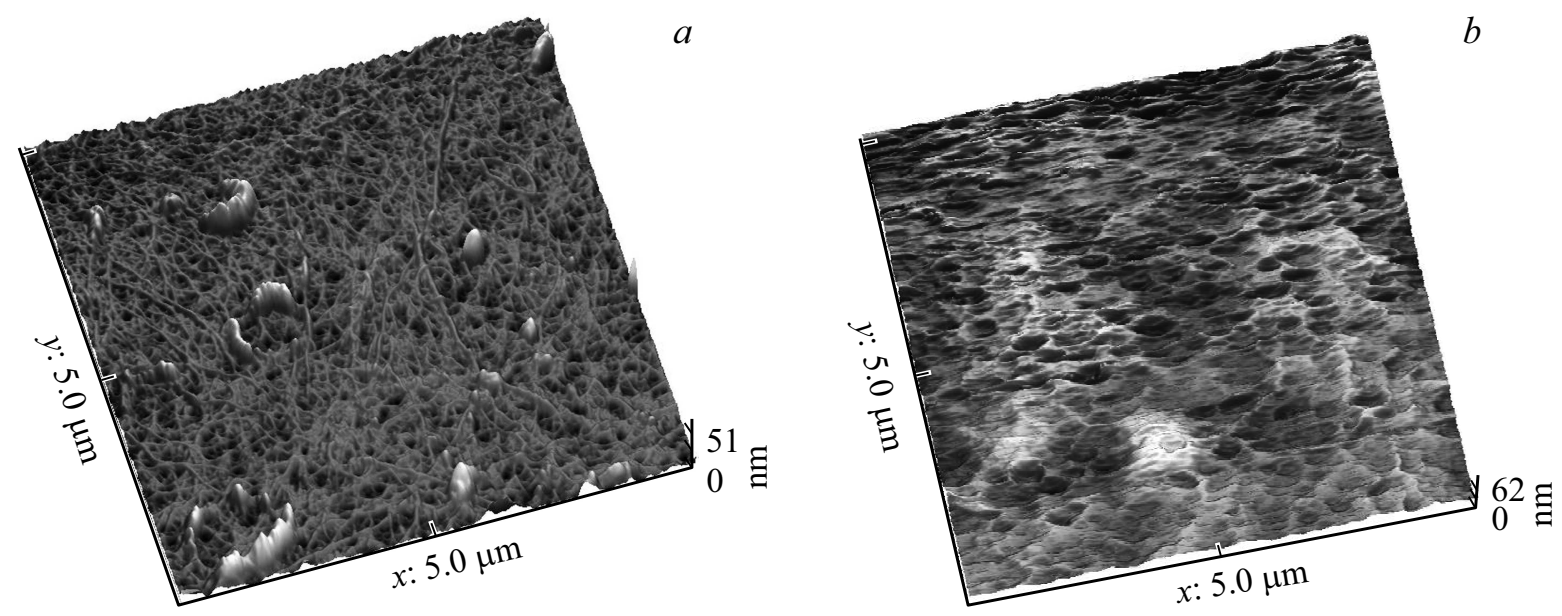

Рис. 1. Изображение поверхности тонкой ОУНТ-пленки с толщиной $11 \pm 3(a)$ и $157 \pm 18(b)$ nm, полученное с помощью атомносиловой микроскопии.

3 $\omega$-метод является достаточно точным методом измерения коэффициента теплопроводности в тонких пленках. В нашем случае, относительная ошибка измерения составляет $\leq 5 \%$. Главный вклад в ошибку измерения вносит ошибка определения температурного коэффициента электрического сопротивления золотой полоски и ошибка определения наклона экспериментальной зависимости $U_{3 \omega}$ от $\operatorname{Ln}(2 \omega)$. Авторы работ [13-15] также отмечали, что относительная ошибка измерения коэффициента теплопроводности с помощью $3 \omega$-метода была не более $2 \%$.

\section{3. Экспериментальные результаты и обсуждение}

Поверхность тонкой пленки на основе одностенных углеродных нанотрубок с толщиной $11 \pm 3$ и $157 \pm 18 \mathrm{~nm}$ представлена на рис. $1, a$ и $b$ соответственно.

На поверхности тонкой ОУНТ-пленки с толщиной $11 \pm 3 \mathrm{~nm}$ различимы отдельные нанотрубки. Также, наблюдается более пористая поверхность по сравнению с $157 \pm 18$ nm ОУНТ-пленкой.

На рис. 2 представлена зависимость $\Delta T$ от $\operatorname{Ln}(2 \omega)$ для 5 образцов тонких ОУНТ-пленок, а также для стеклянной подложки.

Как видно из рис. 2 , только зависимости $\Delta T$ от $\operatorname{Ln}(2 \omega)$ тонких ОУНТ пленок с толщиной $11 \pm 3$ и $34 \pm 12 \mathrm{~nm}$ параллельны относительно зависимости подложки. Это означает, что тепловой поток от золотой полоски проходит через тонкую ОУНТ пленку в подложку. В этом случае коэффициент теплопроводности рассчитывался согласно формуле (2). Интересный эффект наблюдается, когда толщина ОУНТ-пленки составляет $49 \pm 8 \mathrm{~nm}$. На рис. 2. показано, что зависимость $\Delta T$ от $\operatorname{Ln}(2 \omega)$ резко меняет наклон относительно зависимости для подложки. Физически эту ситуацию можно объяснять тем, что

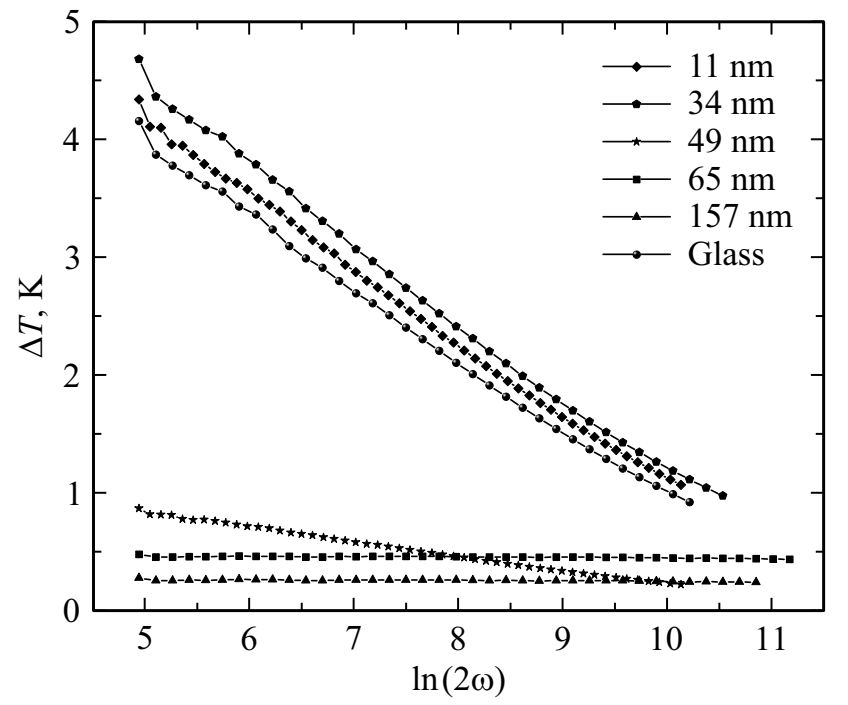

Рис. 2. Зависимость $\Delta T$ от $\operatorname{Ln}(2 \omega)$ для стеклянной подложки и тонких ОУНТ-пленок с толщиной $11,34,49,65$ и $157 \mathrm{~nm}$, полученных с помощью вакуумной фильтрации.

тепловой поток эффективно растекается в плоскость пленки. Исследуемая тонкая ОУНТ-пленка является системой уложенных в плоскость одностенных углеродных нанотрубок. В экспериментальной работе [16] было показано, что коэффициент теплопроводности индивидуальной одностенной углеродной нанотрубки достигает $3500 \mathrm{~W} \cdot \mathrm{m}^{-1} \cdot \mathrm{K}^{-1}$. В конечном счете, система из таких нанотрубок может распределять тепловой поток в плоскость пленки. Однако часть теплового потока может дойти до подложки. Этот случай является самым сложным с точки зрения расчета коэффициента теплопроводности тонкой ОУНТ-пленки, поскольку неизвестно соотношение пройдённого теплового потока в подложку к распределенному тепловому потоку в плоскости. При толщинах $65 \pm 4$ и $157 \pm 18 \mathrm{~nm}$ наклоны зависимостей $\Delta T$ 


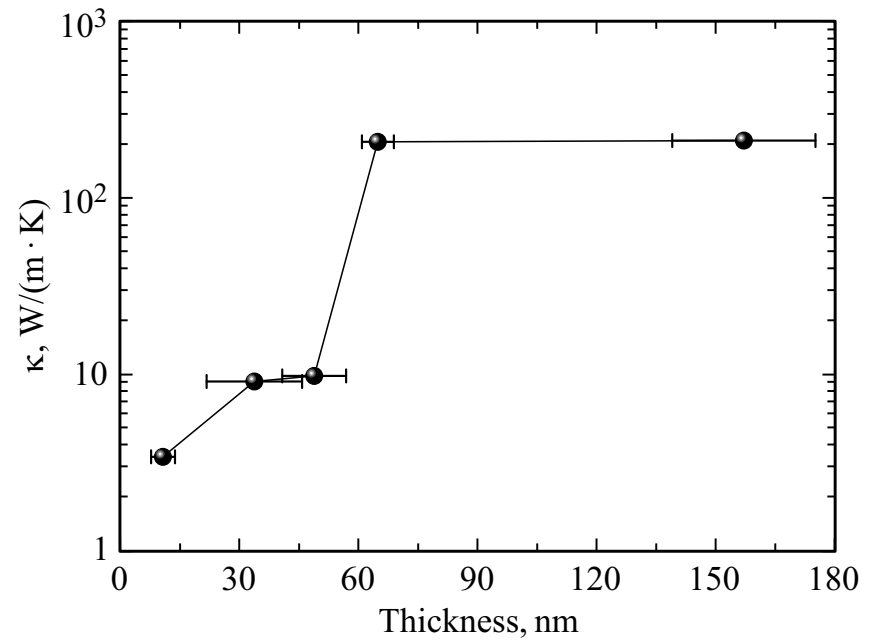

Рис. 3. Зависимость коэффициента теплопроводности от толщины пленки при комнатной температуре.

от $\operatorname{Ln}(2 \omega)$ полностью не совпадают с зависимостью для подложки. В этой ситуации тепловой поток полностью растекается в плоскость тонкой ОУНТ-пленки и не проходит через границу пленка/подложка. Таким образом, для расчета коэффициента теплопроводности в тонких ОУНТ-пленках с толщиной $11 \pm 3$ и $34 \pm 12 \mathrm{~nm}$ использовалась формула 2 , а для пленок с толщиной $49 \pm 8,65 \pm 4$ и $157 \pm 18 \mathrm{~nm}$ использовалась формула 3.

На рис. 3 представлена зависимость коэффициента теплопроводности от толщины для тонких ОУНТпленок.

Из рис. 3 видно, что коэффициент теплопроводности сильно зависит от толщины пленки. При толщине пленки $11 \pm 3 \mathrm{~nm}$ коэффициент теплопроводности составлял $3.42 \pm 0.17 \mathrm{~W} \cdot \mathrm{m}^{-1} \cdot \mathrm{K}^{-1}$. Однако, при толщине $65 \pm 4 \mathrm{~nm}$ коэффициент теплопроводности достигал $208 \pm 10 \mathrm{~W} \cdot \mathrm{m}^{-1} \cdot \mathrm{K}^{-1}$. Отношение коэффициента теплопроводности для $65 \pm 4 \mathrm{~nm}$ к $11 \pm 3 \mathrm{~nm}$ пленке составило $\sim 60$. Дальнейшее увеличение толщины пленки практически не влияет на значение коэффициента теплопроводности. Так, для $157 \pm 18 \mathrm{~nm}$ пленки коэффициент теплопроводности был $211 \pm 11 \mathrm{~W} \cdot \mathrm{m}^{-1} \cdot \mathrm{K}^{-1}$. Нами было найдено всего несколько работ $[17,18]$, в которых было показано, что коэффициент теплопроводности зависит от толщины пленки. В данных работах было обнаружено, что коэффициент теплопроводности увеличивается при увеличении толщины пленки. Однако нами не было обнаружено в научных работах такой сильной зависимости коэффициента теплопроводности от толщины. Возможно, что при малых толщинах тонкие ОУНТ-пленки формируются с большими порами по сравнению с толстыми пленками. Вероятно, что это приводит к снижению коэффициента теплопроводности. Здесь стоит отметить, что для тонкой пленки с толщиной $49 \pm 8 \mathrm{~nm}$ значение коэффициента теплопроводности является заниженным, поскольку часть теплового потока доходит до подложки. При этом расчет проводился по формуле 3 для массивных образцов. Тем не менее, впервые было обнаружено, что критической толщиной тонких ОУНТ-пленок, при которой тепловой поток эффективно растекается в плоскость пленки, и нет зависимости от подложки, является $49 \pm 8 \mathrm{~nm}$.

Температурная зависимость коэффициента теплопроводности представлена на рис. 4.

Коэффициент теплопроводности, как видно из рис. 4, для пленок с толщиной $11 \pm 3$ и $34 \pm 12 \mathrm{~nm}$ относительно слабо зависит от температуры. Начиная с толщины $49 \pm 8 \mathrm{~nm}$ коэффициент теплопроводности начинает увеличиваться с $375 \mathrm{~K}$. При температуре $450 \mathrm{~K}$ коэффициент теплопроводности имел значение $12.6 \pm 0.6 \mathrm{~W} \cdot \mathrm{m}^{-1} \cdot \mathrm{K}^{-1}$. Сильный температурный эффект наблюдался при толщинах $65 \pm 4$ и $157 \pm 18 \mathrm{~nm}$. При увеличении температуры коэффициент тепловодности резко начинал уменьшаться с $211 \pm 11 \mathrm{~W} \cdot \mathrm{m}^{-1} \cdot \mathrm{K}^{-1}$ при $300 \mathrm{~K}$ до $27.5 \pm 1.4 \mathrm{~W} \cdot \mathrm{m}^{-1} \cdot \mathrm{K}^{-1}$ при $450 \mathrm{~K}$. Изменение составило $\sim 8$ раз. Стоит отметить, что значение $211 \pm 11 \mathrm{~W} \cdot \mathrm{m}^{-1} \cdot \mathrm{K}^{-1}$ при $300 \mathrm{~K}$ хорошо коррелирует со значением коэффициента теплопроводности для $5 \mu \mathrm{m}$ пленки ОУНТ [19]. В научной литературе было найдено несколько работ, в которых коэффициент теплопроводности тонких ОУНТ-пленок был измерен при температуре выше комнатной [20,21]. В целом можно отметить схожую температурную зависимость коэффициента теплопроводности для тонких ОУНТ-пленок. Однако, такого сильного температурного эффекта как для $65 \pm 4$ и $157 \pm 18 \mathrm{~nm}$ пленок не было обнаружено. Одним из возможных объяснений такой температурной зависимости коэффициента теплопроводности может быть большое фононное рассеяние на границе между нанотрубками [20].

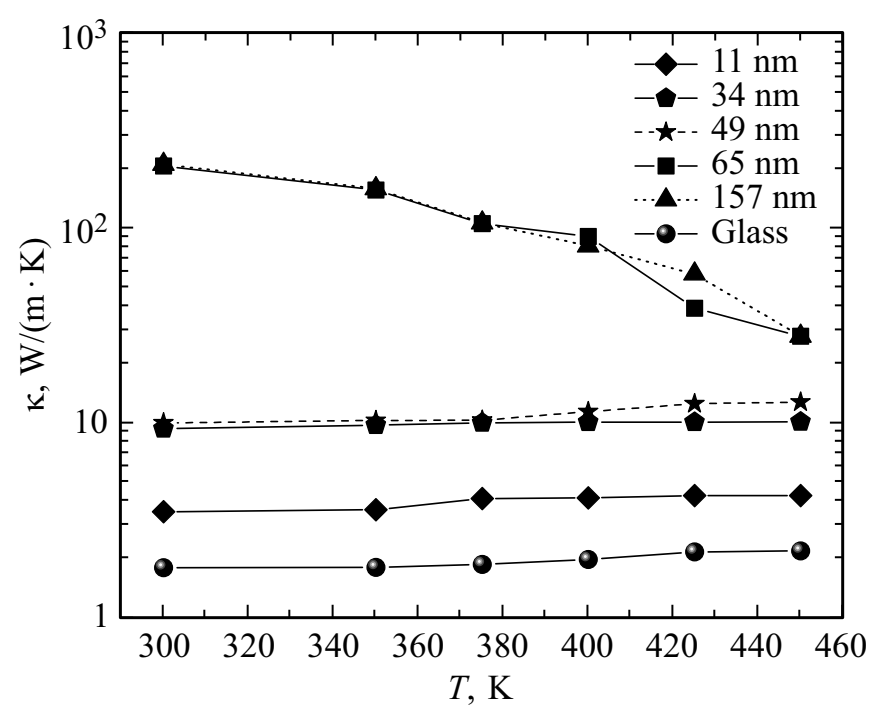

Рис. 4. Зависимость коэффициента теплопроводности от температуры для тонких ОУНТ-пленок с толщиной 11, 34, 49, 65 и $157 \mathrm{~nm}$. 
Таким образом, полученные в настоящей работе зависимости коэффициента теплопроводности от толщины и температуры могут быть полезны для разработки гибкой электроники и термоэлектрических преобразователей на основе ОУНТ.

\section{4. Заключение}

В настоящей работе были сформированы тонкие ОУНТ-пленки толщиной $11 \pm 3,34 \pm 12,49 \pm 8,65 \pm 4$ и $157 \pm 18 \mathrm{~nm} \mathrm{c} \mathrm{помощью} \mathrm{вакуумной} \mathrm{фильтрации.} \mathrm{Для}$ измерения коэффициента теплопроводности в тонких ОУНТ-пленках использовался $3 \omega$-метод. Температурные измерения коэффициента теплопроводности были проведены в температурном диапазоне $300 \pm 450 \mathrm{~K}$. Впервые была обнаружена переходная толщина в области $49 \mathrm{~nm}$ для тонких ОУНТ-пленок, при которой коэффициент теплопроводности измерялся без учета подложки. Обнаружена сильная зависимость коэффициента теплопроводности от толщины. Изменение толщины с $11 \pm 3$ до $65 \pm 4 \mathrm{~nm}$ приводило к увеличению коэффициента теплопроводности примерно в 60 раз. Сильный температурный эффект наблюдался для $65 \pm 4$ и $157 \pm 18 \mathrm{~nm}$ пленок. Коэффициент теплопроводности $157 \pm 18 \mathrm{~nm}$ пленки стремительно уменьшался с $211 \pm 11 \mathrm{~W} \cdot \mathrm{m}^{-1} \cdot \mathrm{K}^{-1}$ при $300 \mathrm{~K}$ до $27.5 \pm 1.4 \mathrm{~W} \cdot \mathrm{m}^{-1} \cdot \mathrm{K}^{-1}$ при $450 \mathrm{~K}$. Изменение составило $\sim 8$ раз. Найденные зависимости коэффициента теплопроводности от толщины и температуры могут быть использованы при разработке гибкой электроники и термоэлектрических преобразователей на основе ОУНТ.

\section{Благодарности}

Электронно-микроскопические исследования выполнены в Красноярском региональном центре коллективного пользования ФИЦ КНЦ СО РАН.

\section{Финансирование работы}

Исследование выполнено при финансовой поддержке Российского фонда фундаментальных исследований, Правительства Красноярского края, Красноярского краевого фонда науки в рамках научного проекта: „Исследование коэффициента теплопроводности и структурных особенностей в тонких наноструктурированных оксидных пленках, перспективных для термоэлектрического применения“ (проект № 18-42-243010) и стипендии Президента Российской Федерации (СП-2235.2019.1).

\section{Список литературы}

[1] S. Park, M. Vosguerichian, Z.A. Bao. Nanoscale 5, 1727 (2013).

[2] T. Cheng, Y.Z. Zhang, W.Y. Lai, W. Huang. Adv. Mater. 27, 3349 (2015).

[3] I.A. Tambasov, A.S. Voronin, N.P. Evsevskaya, M.N. Volochaev, Y.V. Fadeev, A.S. Krylov, A.S. Aleksandrovskii, A.V. Luk'yanenko, S.R. Abelyan, E.V. Tambasova. Phys. Solid State 60, 2649 (2018).

[4] J.L. Blackburn, A.J. Ferguson, C. Cho, J.C. Grunlan. Adv. Mater. 30, 1704386 (2018).

[5] A.V. Eletskii. Phys. Usp. 52, 209 (2009).

[6] E. Brown, L. Hao, J.C. Gallop, J.C. Macfarlane. Appl. Phys. Lett. 87, 023107 (2005).

[7] A. Kamyshny, S. Magdassi. Chem. Soc. Rev. 48, 1712 (2019).

[8] B. Kumanek, D. Janas. J. Mater. Sci. 54, 7397 (2019).

[9] A.D. Avery, B.H. Zhou, J. Lee, E.S. Lee, E.M. Miller, R. Ihly, D. Wesenberg, K.S. Mistry, S.L. Guillot, B.L. Zink, Y.H. Kim, J.L. Blackburn, A.J. Ferguson. Nature Energy 1, 16033 (2016).

[10] I.A. Tambasov, A.S. Voronin, N.P. Evsevskaya, M.N. Volochaev, Y.V. Fadeev, M.M. Simunin, A.S. Aleksandrovsky, T.E. Smolyarova, S.R. Abelian, E.V. Tambasova, M.O. Gornakov, V.A. Eremina, Y.M. Kuznetsov, M.V. Dorokhin, E.D. Obraztsova. Physica E 114, 113619 (2019).

[11] I.A. Tambasov, M.N. Volochaev, A.S. Voronin, N.P. Evsevskaya, A.N. Masyugin, A.S. Aleksandrovskii, T.E. Smolyarova, I.V. Nemtsev, S.A. Lyashchenko, G.N. Bondarenko, E.V. Tambasova. Phys. Solid State 61, 1904 (2019).

[12] D.G. Cahill, M. Katiyar, J.R. Abelson. Phys. Rev. B 50, 6077 (1994).

[13] D.G. Cahill. Rev. Sci. Instrum. 61, 802 (1990).

[14] F. Chen, J. Shulman, Y. Xue, C.W. Chu, G.S. Nolas. Rev. Sci. Instrum. 75, 4578 (2004).

[15] D.W. Oh, A. Jain, J.K. Eaton, K.E. Goodson, J.S. Lee. Int. J. Heat Fluid Fl 29, 1456 (2008).

[16] E. Pop, D. Mann, Q. Wang, K.E. Goodson, H.J. Dai. Nano Lett. 6, 96 (2006).

[17] S. Yoshida, Y. Feng, C. Delacou, T. Inoue, R. Xiang, R. Kometani, S. Chiashi, E.I. Kauppinen, S. Maruyama. Nanotechnology 28, 185701 (2017).

[18] Y. Feng, T. Inoue, M. Watanabe, S. Yoshida, Y. Qian, R. Xiang, E.I. Kauppinen, S. Chiashi, S. Maruyama. Jpn J. Appl. Phys. 57, 075101 (2018).

[19] J. Hone, M.C. Llaguno, N.M. Nemes, A.T. Johnson, J.E. Fischer, D.A. Walters, M.J. Casavant, J. Schmidt, R.E. Smalley. Appl. Phys. Lett. 77, 666 (2000).

[20] A. Duzynska, A. Taube, K.P. Korona, J. Judek, M. Zdrojek. Appl. Phys. Lett. 106, 183108 (2015).

[21] M. Yarali, J. Hao, M. Khodadadi, H. Brahmi, S. Chen, V.G. Hadjiev, Y.J. Jung, A. Mavrokefalos. Rsc Advances 7, 14078 (2017).

Редактор Д.В. Жуманов

\section{Конфликт интересов}

Авторы заявляют, что у них нет конфликта интересов. 